Check for updates

Cite this: Mater. Adv., 2021, 2, 2711

Received 29th January 2021, Accepted 18th March 2021

DOI: $10.1039 / \mathrm{d} 1 \mathrm{ma} 00085 \mathrm{c}$

rsc.li/materials-advances

\section{Free-standing bilayered vanadium oxide films synthesized by liquid exfoliation of chemically preintercalated $\delta-\mathrm{Li}_{x} \mathrm{~V}_{2} \mathrm{O}_{5} \cdot n \mathrm{H}_{2} \mathrm{O} \dagger$}

\author{
Luke Houseman, ${ }^{a}$ Santanu Mukherjee, ${ }^{a}$ Ryan Andris, (D) ${ }^{a}$ Michael J. Zachman ${ }^{\mathrm{b}}$ and \\ Ekaterina Pomerantseva (iD *a
}

\begin{abstract}
A free-standing film composed of bilayered vanadium oxide nanoflakes is for the first time synthesized using a new low-energy process. The precursor powder, $\delta-\mathrm{Li}_{x} \mathrm{~V}_{2} \mathrm{O}_{5} \cdot n \mathrm{H}_{2} \mathrm{O}$, was prepared using a simple sol-gel based chemical preintercalation synthesis procedure. $\delta-\mathrm{Li}_{x} \mathrm{~V}_{2} \mathrm{O}_{5} \cdot n \mathrm{H}_{2} \mathrm{O}$ was dispersed and probe sonicated in $\mathrm{N}$-methyl pyrrolidone to exfoliate the bilayers followed by vacuum filtration resulting in the formation of a free-standing film with obsidian color. X-ray diffraction showed lamellar ordering of a single-phase material with a decreased interlayer distance compared to that of the precursor powder. Scanning electron microscopy images demonstrated stacking of the individual nanoflakes. This morphology was further confirmed with scanning transmission electron microscopy that showed highly malleable nanoflakes consisting of $\sim 10-100$ vanadium oxide bilayers. One of the most important consequences of this morphological rearrangement is that the electronic conductivity of the freestanding film, measured by the four-probe method, increased by an order of magnitude compared to conductivity of the pressed pellet made of precursor powder. X-ray photoelectron spectroscopy measurements showed the coexistence of both $\mathrm{V}^{5+}$ and $\mathrm{V}^{4+}$ oxidation states in the exfoliated sample, possibly contributing to the change in electronic conductivity. The developed approach provides the ability to maintain the phase purity and crystallographic order during the exfoliation process, coupled with the formation of a free-standing film of enhanced conductivity. The produced bilayered vanadium oxide nanoflakes can be used as the building blocks for the synthesis of versatile two-dimensional heterostructures to create innovative electrodes for electrochemical energy storage applications.
\end{abstract}

\section{Introduction}

Two-dimensional (2D) materials with layered structures have drawn considerable research attention due to their unique and often enhanced properties as compared to their bulk precursors and conventional materials. ${ }^{1-4}$ As a manifestation of their nanoflake morphology and layered structure, these materials demonstrate high surface area and 2D ion diffusion channels that provide greater access to electrochemically active sites and improved ion transfer kinetics. ${ }^{5,6}$ In addition, their interlayer regions can be easily accessed and modulated, thereby making these materials highly suitable for intercalation driven applications, such as electrochemical energy storage. ${ }^{2,7-9}$ A library of $2 \mathrm{D}$

\footnotetext{
${ }^{a}$ Department of Materials Science and Engineering, Drexel University, Philadelphia, PA, 19104, USA.E-mail: ep423@drexel.edu

${ }^{b}$ Center for Nanophase Materials Sciences, Oak Ridge National Laboratory, Oak Ridge, TN 37831, USA

$\dagger$ Electronic supplementary information (ESI) available. See DOI: 10.1039/ d1ma00085c
}

materials with a spectrum of structures and chemistries such as graphene, transition metal dichalcogenides, oxides and carbides (MXenes) have found widespread usage as electrodes in batteries and supercapacitors. ${ }^{4,7,10,11}$

One of the most important and prolific techniques for the synthesis of 2D materials from layered bulk precursors is liquid exfoliation. ${ }^{12,13}$ In this process, a top-down approach, the bulk precursor is sonicated in a liquid solvent (e.g., alcohols or butyllithium), sometimes in the presence of intercalating species, which results in the "shearing" or delamination of the constituent layers of the bulk material. ${ }^{14-16}$ The individual layers obtained from the liquid exfoliation process can then be reassembled in sophisticated structures. This process can be achieved using organic molecules (e.g., dopamine), inorganic ions (e.g., $\mathrm{Li}^{+}, \mathrm{Na}^{+}$), or even by combining the layers with dissimilar building blocks in a single architecture to create a heterostructure. ${ }^{17-19}$ This liquid exfoliation technique also enables size-control and anti-oxidation characteristics to the exfoliated sheets, which can facilitate electrochemical performance. ${ }^{20,21}$ 
However, most of the layered materials, including graphene, TMDs, and MXenes, serve as anode materials. Therefore, it is necessary to develop reliable and high-quality 2D cathode materials to build next-generation energy storage devices by advanced nanomanufacturing approaches. ${ }^{22}$ Vanadium oxide is a particularly interesting model material for this purpose. ${ }^{23}$ The most common vanadium oxide phase, orthorhombic $\alpha-\mathrm{V}_{2} \mathrm{O}_{5}$, has drawn considerable interest as a positive electrode material. It has a high theoretical capacity, is readily available, and economically viable. The structure of $\alpha-\mathrm{V}_{2} \mathrm{O}_{5}$ consists of layers built by monolayers of $\mathrm{VO}_{5}$ square pyramids with an interlayer distance of $4.4 \AA$. Another polymorph, bilayered (or $\delta$-) vanadium oxide, attracts attention due to the specifics of its structure and chemical composition. The distance between the centers of $\mathrm{V}-\mathrm{O}$ layers in the parent bilayered vanadium oxide is $11.5 \AA$, and this large interlayer spacing is stabilized by water molecules; therefore the chemical composition of this material is usually represented as $\delta-\mathrm{V}_{2} \mathrm{O}_{5} \cdot n \mathrm{H}_{2} \mathrm{O}$. Advances in materials chemistry, enabled by the through-synthesis incorporation of inorganic ions and water molecules into the interlayer region, led to the formation of a large family of bilayered vanadium oxides with chemical compositions of $\delta-\mathrm{M}_{x} \mathrm{~V}_{2} \mathrm{O}_{5} \cdot n \mathrm{H}_{2} \mathrm{O}$ $(\mathrm{M}=\mathrm{Li}, \mathrm{Na}, \mathrm{K}, \mathrm{Mg}, \mathrm{Ca}, \mathrm{Zn}, \mathrm{Mn}, \mathrm{Ni}$ ) and tunable interlayer spacing. ${ }^{24-30}$ As the name suggests, $\mathrm{V}-\mathrm{O}$ layers are built by two rows of edge-sharing $\mathrm{VO}_{6}$ octahedra units. ${ }^{31}$ As a result, compared to the $\alpha-\mathrm{V}_{2} \mathrm{O}_{5}$ structure, the layers in bilayered vanadium oxide are characterized by the increased number of redox active centers per layer. Indeed, previous research on alkali ion preintercalated layered titanates revealed that $\mathrm{Na}$ - and K-containing materials with the structural $\mathrm{Ti}-\mathrm{O}$ layers built by two $\mathrm{Ti}$ rows exhibited much higher electrochemical activity when used as electrodes in cells with aqueous $\mathrm{Na}_{2} \mathrm{SO}_{4}$ electrolyte compared to the Li-containing phase with the Ti-O layers composed of a single straight layer of $\mathrm{Ti}$ atoms in octahedral oxygen configuration. ${ }^{32}$ Alkali and alkaline-earth metal ion preintercalated bilayered $\mathrm{V}_{2} \mathrm{O}_{5}$ with tunable interlayer spacings ranging from 9.6 to $13.4 \AA$ can be synthesized using a sol-gel based approach. ${ }^{26,33-35}$

Liquid exfoliation of $\alpha-\mathrm{V}_{2} \mathrm{O}_{5}$ was reported to produce electrochemically active nanosheets. For example, large amounts of vanadium oxide nanosheets were obtained by sonicating commercial $\alpha-\mathrm{V}_{2} \mathrm{O}_{5}$ in both water and ethanol, where each solvent resulted in different interlayer spacings. ${ }^{36}$ Similarly, Rui et al. used a simple sonication based technique in formamide solvent to obtain $\alpha-\mathrm{V}_{2} \mathrm{O}_{5}$ nanosheets, achieving a thickness of $2.1-3.8 \mathrm{~nm}$ in the process. ${ }^{37}$ Likewise, a hydrolysis based approach was utilized by Diem $e t$ al. to successfully synthesize self-supporting vanadium oxide nanofiber films demonstrating reasonable electrochemical activity without any conductive agent. ${ }^{38}$ Etman et al. have also used aqueous media to synthesize and exfoliate $\mathrm{V}_{2} \mathrm{O}_{5}$ nanosheets using a reflux based technique with $\alpha-\mathrm{V}_{2} \mathrm{O}_{5}$ and $\mathrm{VO}_{2}$ as precursor materials. ${ }^{39,40}$ However, to the best of our knowledge, bilayered vanadium oxides have not yet been used as an exfoliation precursor.

In this manuscript, we report a simple, low energy technique to exfoliate bilayered vanadium oxide and obtain stable nanoflake suspensions. The $\delta-\mathrm{Li}_{x} \mathrm{~V}_{2} \mathrm{O}_{5} \cdot n \mathrm{H}_{2} \mathrm{O}$ phase has been selected as a precursor because it has the largest interlayer distance among alkali metal ion preintercalated bilayered vanadium oxides and singly-charged nature of the interlayer $\mathrm{Li}^{+}$ ions. ${ }^{26}$ Vacuum filtration of the suspensions leads to formation of free-standing films composed of stacked bilayered vanadium oxide nanoflakes. The flakes range from a few $\mathrm{nm}$ to approximately $100 \mathrm{~nm}$ in thickness and are flexible, forming intricate curved patterns in the film architecture. The enhanced electronic conductivity of the film as compared to the pressed pellets made of precursor material is advantageous for energy storage applications. The developed exfoliation process combined with nanomanufacturing approaches has a potential to produce flexible electrodes and $2 \mathrm{D}$ heterostructures with advanced functionalities. ${ }^{22,41}$

\section{Experimental procedure}

\subsection{Synthesis}

2.1.1 Synthesis of $\delta-\mathrm{Li}_{x} \mathbf{V}_{2} \mathrm{O}_{5} \cdot \mathbf{n H}_{2} \mathrm{O}$ precursor powder. The synthesis of $\delta-\mathrm{Li}_{x} \mathrm{~V}_{2} \mathrm{O}_{5} \cdot n \mathrm{H}_{2} \mathrm{O}$ (hereafter called "precursor powder") was adopted from a previous report. ${ }^{26}$ In summary, a stoichiometric amount of $\mathrm{LiCl}$ (99\%, Fischer Scientific ${ }^{\mathrm{TM}}$ ) was dissolved in $15 \mathrm{ml}$ of DI water to which $15 \mathrm{ml}$ of $30 \% \mathrm{H}_{2} \mathrm{O}_{2}$ solution (Fischer Scientific $^{\mathrm{TM}}$ ) was subsequently added with simultaneous stirring. This step was followed by the slow addition of $500 \mathrm{mg}$ of $\alpha-\mathrm{V}_{2} \mathrm{O}_{5}$ powder $\left(99 \%\right.$, Fischer Scientific $\left.{ }^{\mathrm{TM}}\right)$. An initial orangecolored transparent solution is obtained indicating the complete dissolution of the $\alpha-\mathrm{V}_{2} \mathrm{O}_{5}$ powder and the formation of peroxovanadate complexes. After two hours, the temperature was raised to $60{ }^{\circ} \mathrm{C}$ and the solution was stirred for another 3 hours that resulted in a gradual color change to deep red. This was followed by a 4-day aging process at room temperature, vacuum filtration, and drying in air at $105{ }^{\circ} \mathrm{C}$ overnight.

2.1.2 Exfoliation of $\delta-\mathbf{L i}_{x} \mathbf{V}_{2} \mathbf{O}_{5} \cdot \mathbf{n H}_{2} \mathbf{O}$ powder. $250 \mathrm{mg}$ of the precursor powder was placed in a $100 \mathrm{ml}$ vial to which $30 \mathrm{ml}$ of $N$-methyl-2-pyrrolidone (NMP) was added. A uniform mixture, created using a vortex mixer, was subsequently subjected to probe sonication (power rating of $125 \mathrm{~W}$ ) for a total duration of 30 minutes. The probe amplitude was $80 \%$, the sonication frequency was $20 \mathrm{kHz}$, and cycles consisted of pulses of 2 seconds on and 2 seconds off for a total cycle time of 1 hour. At the completion of sonication, the contents of the vial were decanted and centrifuged three times, each at $1500 \mathrm{rpm}$ for a duration of 15 minutes. After the completion of each centrifugation cycle, the supernatant was removed and collected. These supernatant fractions were mixed and vacuum filtered using an alumina filter $(0.2 \mu \mathrm{m}$ pore size $)$. At the completion of filtration, a film with obsidian color was collected on the filter surface and dried at $70{ }^{\circ} \mathrm{C}$ in air for 4 hours. Next, it was gently peeled using a razor blade. A schematic of the exfoliation process is shown in Fig. 1.

2.1.2 Preparation of samples for conductivity measurement. The electronic conductivity of the precursor was calculated using the measured sheet resistance of pressed powder pellets. The pellets were prepared using $\sim 10 \mathrm{mg}$ of powder to fill a 

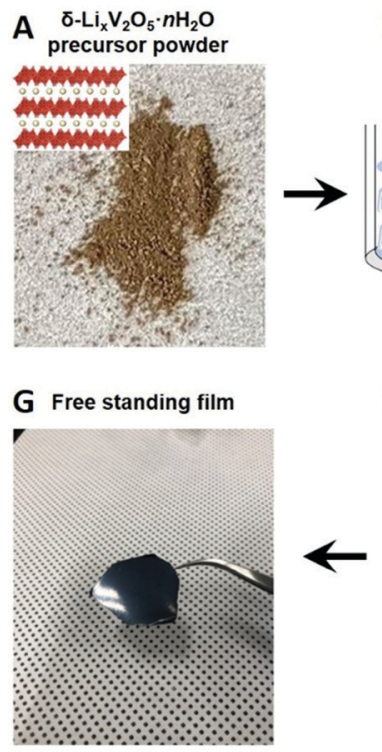

B

\section{C}

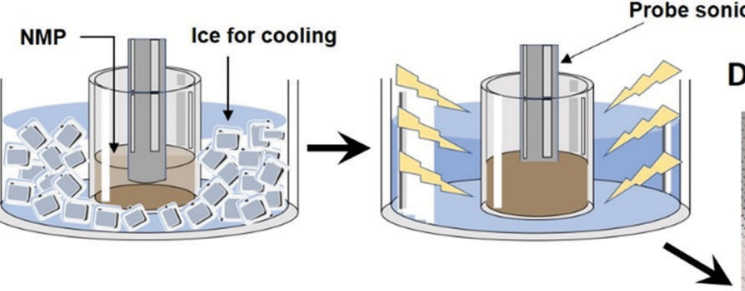

Centrifuged 3 times at 1500

rpm for 15 minutes in NMP

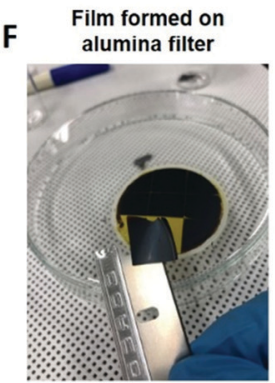

E
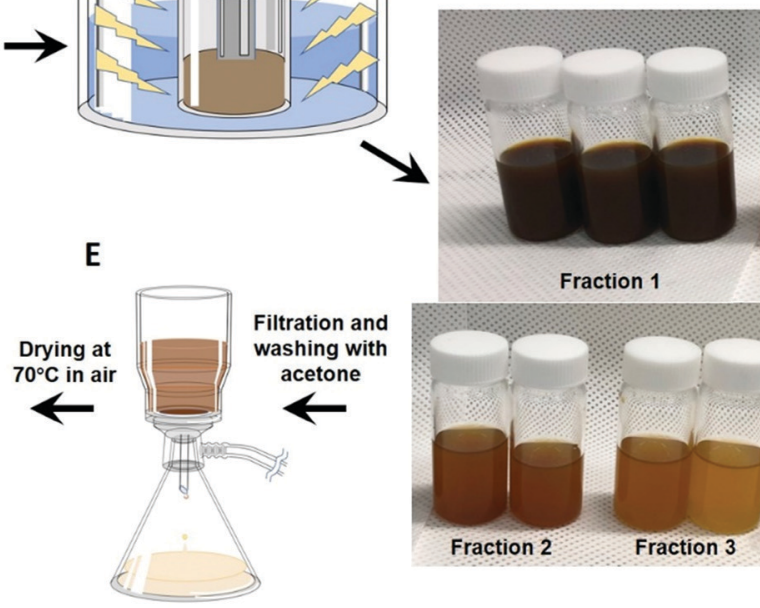

Fraction 1

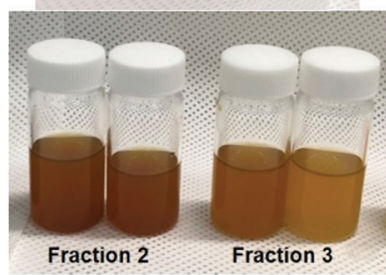

Fig. 1 Schematic illustration of the exfoliation process starting from the $\delta-\mathrm{Li}_{x} \mathrm{~V}_{2} \mathrm{O}_{5} \cdot n \mathrm{H}_{2} \mathrm{O}$ precursor powder. $(\mathrm{A}) \mathrm{A}$ photograph of the $\delta$ - $\mathrm{Li} \mathrm{i}_{x} \mathrm{~V}_{2} \mathrm{O}_{5} \cdot n \mathrm{H}_{2} \mathrm{O}$ precursor powder (the inset shows the structure of bilayered vanadium oxide; yellow spheres represent stabilizing $\mathrm{Li}^{+}$ions and water molecules residing in the interlayer region, while their positions are not specified). (B) Precursor powder suspended in NMP. (C) Probe sonication of NMP-dispersed precursor yielding (D) suspensions of exfoliated nanoflakes. (E) Vacuum filtration of the produced suspensions. (F) A photograph of the film formed on the surface of a filter. (G) A photograph of the free-standing film.

stainless-steel die. The die was placed in a Carver ${ }^{\mathrm{TM}}$ hydraulic press for approximately $10-15$ seconds under $10000 \mathrm{kPa}$. The electronic conductivity of the free-standing film was calculated using the measured sheet resistance of a piece of film. A pictorial representation of the steps involved to prepare each sample for the sheet resistance measurement is provided in Fig. S1 (ESI $\dagger$ ).

\subsection{Materials characterization}

Phase characterization of the materials was carried out using a Rigaku SmartLab X-Ray diffractometer with $\mathrm{Cu}-\mathrm{K} \alpha$ radiation and a $0.02^{\circ}$ step size. The distance between the centers of $\mathrm{V}-\mathrm{O}$ layers ( $d$-spacing) was calculated from the position of the (001) reflection using Bragg's equation. The bulk material morphology was determined using a Zeiss Supra 50 VP scanning electron microscopy (SEM) instrument at a $5 \mathrm{kV}$ accelerating voltage and a $4 \mathrm{~mm}$ working distance. To obtain the cross-sectional SEM images, the free-standing film was partially cut with a razor blade and further ripped apart. Scanning transmission electron microscopy (STEM) characterization was performed on a Nion UltraSTEM 100 at an accelerating voltage of $100 \mathrm{kV}$, a convergence semiangle of $\sim 31 \mathrm{mrad}$, and a beam current of approximately $45 \mathrm{pA}$. Bright-field (BF)-STEM images were acquired using a dose of approximately $6 \times 10^{3} \mathrm{e}^{-} \AA^{-2}$, below the damage threshold for the material, which was determined to be $\sim 10^{4} \mathrm{e}^{-} \AA^{-2}$ by tracking loss of atomic-scale structure in image series. Local layer orientation mapping was performed by taking a series of fast Fourier transforms (FFT) in small windows across the BF-STEM images, identifying the angle and intensity of the FFT peaks arising from the material lattice in the image, and visualizing them in a stream plot. All data was processed in Python using standard packages. Cross-sectional STEM samples were prepared by standard focused ion beam (FIB) techniques. Samples were stored in a vacuum desiccator at all times other than during the FIB preparation and STEM experiment. The interlayer water content of the materials was measured with a Q50 thermogravimetric analyzer. The degree of hydration was determined by evaluating the weight loss within the temperature range of $30-600{ }^{\circ} \mathrm{C}$ in air, following previous findings about temperature range relevant to the evaporation of interlayer water molecules in bilayered vanadium oxides. ${ }^{35} \mathrm{X}$-ray photoelectron spectroscopy (XPS) was used to identify the changes in the oxidation state of vanadium during the exfoliation process. Measurements were recorded on a Physical Electronics VersaProbe 5000 using a monochromatic Al K $\alpha$ source and charge compensation. The high-resolution $\mathrm{V}_{2 \mathrm{p} 3}$ spectra were taken at a pass energy of $23.5 \mathrm{eV}$ with a step size of $0.05 \mathrm{eV}$. Peak fitting and data analysis were carried out using Casa XPS software. A Shirley background subtraction was used for $\mathrm{V}_{2 \mathrm{p} 3}$ spectra quantification. The sheet resistance was measured using a Jandel instrument utilizing a four-point probe technique. The sheet resistance of the precursor powder and free-standing film was converted to conductivity using eqn (1), where $\sigma$ is the conductivity, $R_{\mathrm{S}}$ is the sheet resistance, and $t$ is the thickness of the pellet or film.

$$
\sigma=\frac{1}{R_{\mathrm{s}} \times t}
$$

\section{Results and discussion}

XRD patterns of the precursor powder and the free-standing film are shown in Fig. 2A. Both samples exhibit a series of (00l) reflections with the prominent (001) peak indicative of the 

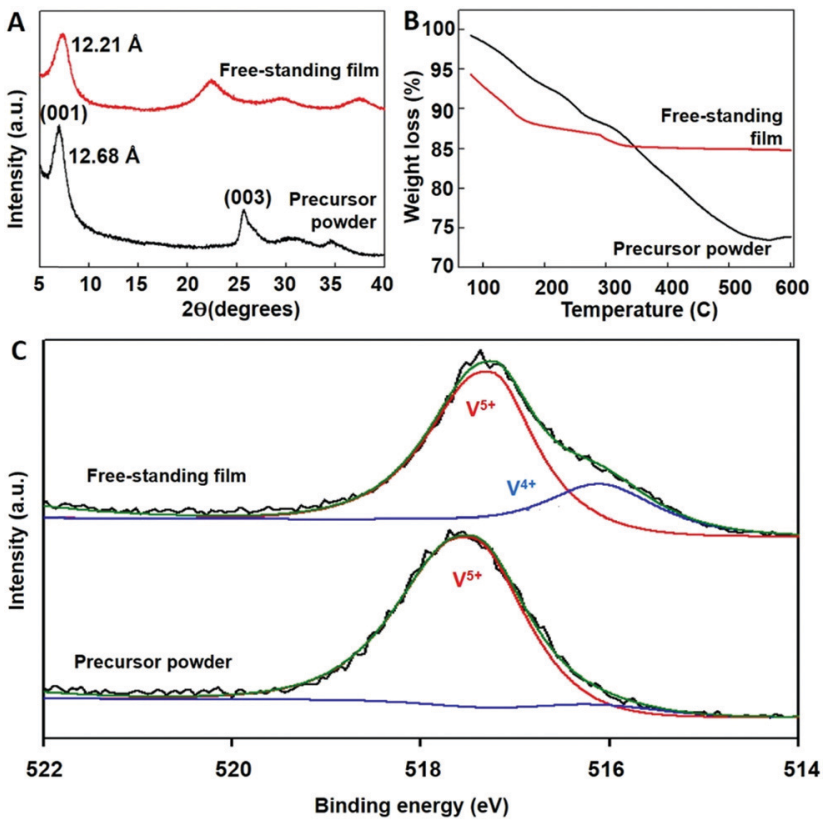

Fig. 2 Phase and chemical composition characterization of the precursor powder and free-standing film. (A) XRD patterns, (B) TGA weight loss curves and (C) XPS spectra of the $V 2 p_{3 / 2}$ core level of the $\delta-L i_{x} V_{2} \mathrm{O}_{5}$. $n \mathrm{H}_{2} \mathrm{O}$ precursor powder and free-standing film prepared by liquid exfoliation of the precursor followed by vacuum filtration.

lamellar ordering. However, the exfoliated sample demonstrates a slightly smaller $d$-spacing (12.21 $\AA$ ) compared to the precursor powder $(12.68 \AA)$. This result can largely be attributed to the rearrangement of the layers during exfoliation/re-assembly process combined with the variability of the chemical composition in the interlayer region. The amount of crystallographic water as well as preintercalated $\mathrm{Li}^{+}$ions can change during the exfoliation/drying process. Aside from the peak shift, only ( $00 l$ reflections are seen in both the bulk precursor and the free-standing film, indicating that the exfoliation process did not result in phase disintegration or the formation of secondary phases.

Thermogravimetric analysis (Fig. 2B) was used to estimate thermal stability and weight loss of the pristine $\delta-\mathrm{Li}_{x} \mathrm{~V}_{2} \mathrm{O}_{5} \cdot n \mathrm{H}_{2} \mathrm{O}$ precursor and free-standing film obtained by exfoliation in NMP followed by vacuum filtration. The weight loss between $100{ }^{\circ} \mathrm{C}$ and $600{ }^{\circ} \mathrm{C}$ corresponds to the loss of interlayer water. ${ }^{35}$ The bulk precursor sample exhibits an approximately $25 \%$ weight reduction compared to an $8.7 \%$ reduction demonstrated by the free-standing film. This result can be attributed to the higher interlayer structural water content in the bulk precursor as compared to the free-standing film, which is in agreement with the (001) peak shift in the XRD pattern. ${ }^{42-44}$ It's likely that the free-standing film has a lower degree of hydration because the exfoliation process occurs in NMP.

X-ray photoelectron spectroscopy (XPS) was used to identify changes in the vanadium oxidation state during the exfoliation process. The XPS survey scans are shown in Fig. S2 (ESI $\dagger$ ). Fig. 2C shows the $\mathrm{V}_{2 \mathrm{p} 3 / 2}$ high resolution spectra for the precursor and free-standing film. Both scans were deconvoluted into peaks located at approximately $517 \mathrm{eV}$ and $516 \mathrm{eV}$, corresponding to $\mathrm{V}^{5+}$ and $\mathrm{V}^{4+}$ valence states, respectively. The vanadium in the precursor powder is predominately in a $5^{+}$ oxidation state. In contrast, there is significant amount of $\mathrm{V}^{4+}$ in the free-standing film. Similar phenomenon of vanadium reduction post exfoliation process has been previously observed for $\alpha-\mathrm{V}_{2} \mathrm{O}_{5}$ phase. ${ }^{36}$ The reduction in vanadium could be attributed to an increase in oxygen vacancies formed during the exfoliation process. ${ }^{45}$

To directly examine the structure of the film, we turned to electron microscopy. Fig. 3(A and B) shows SEM images of the top view of the precursor powder and free-standing film, respectively. The precursor powder exhibits a crumpled sheetlike morphology. A notable change in appearance is observed upon exfoliation, revealing a more planar surface structure. Cross-sectional SEM images of the film (Fig. 3(C and D)) demonstrate multiple layers that are stacked uniformly one above the other, with lateral flake dimensions of hundreds of nanometers or more (Fig. 3B). The distribution of the layers can be observed in greater detail in Fig. S3 (ESI $\dagger$ ).

Additional details about the morphology of the flakes within the free-standing film were obtained by aberration-corrected BF-STEM imaging, as shown in Fig. 4. The alternating light and dark layers in these images originate from the interlayer space and vanadium oxide bilayer lattice, respectively. Fig. 4A shows a representative image of the atomic-scale structure of a flake, which typically contains on the order of 10-100 vanadium bilayers. Fast Fourier transforms (FFTs) of images spanning hundreds of nanometers, as shown in Fig. 4B, reveal that flakes are generally oriented in a consistent direction, but with a fairly broad distribution of local orientations, suggesting a variety of local flake structures are present. The FFTs additionally provide the mean bilayer spacing across this field of view, which was $9.9 \AA$ in the region imaged for Fig. 4B. The discrepancy in $d$-spacing values measured through the XRD and TEM characterization can be related to bulk and local nature of the measurements,
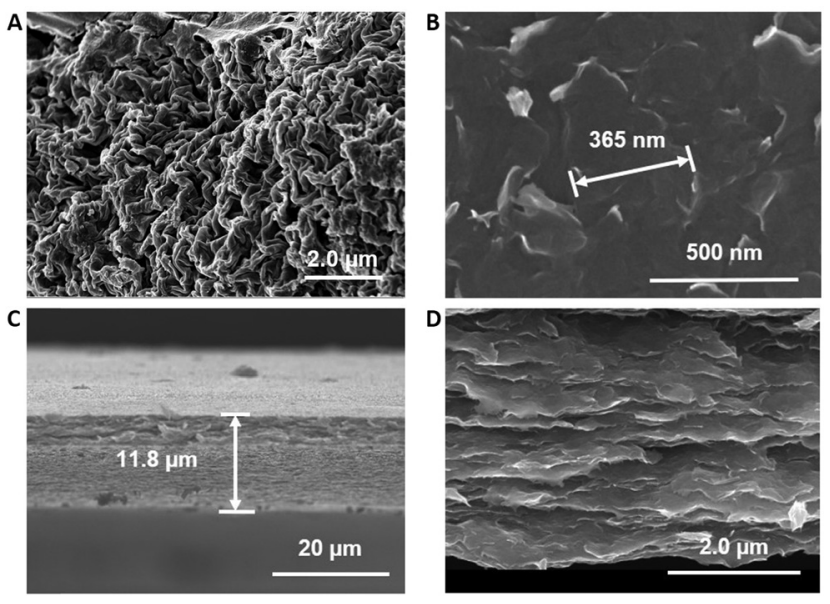

Fig. 3 Morphology characterization of the pristine and exfoliated bilayered vanadium oxides. Top view SEM images of $(A)$ the $\delta-\mathrm{Li}_{x} \mathrm{~V}_{2} \mathrm{O}_{5}$. $n \mathrm{H}_{2} \mathrm{O}$ precursor powder and (B) free-standing film. (C and D) Cross-sectional SEM images of the free-standing film obtained via liquid exfoliation process followed by vacuum filtration. 
A

A

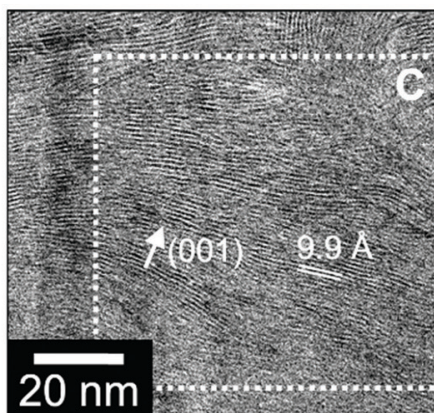

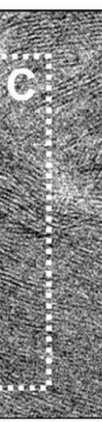
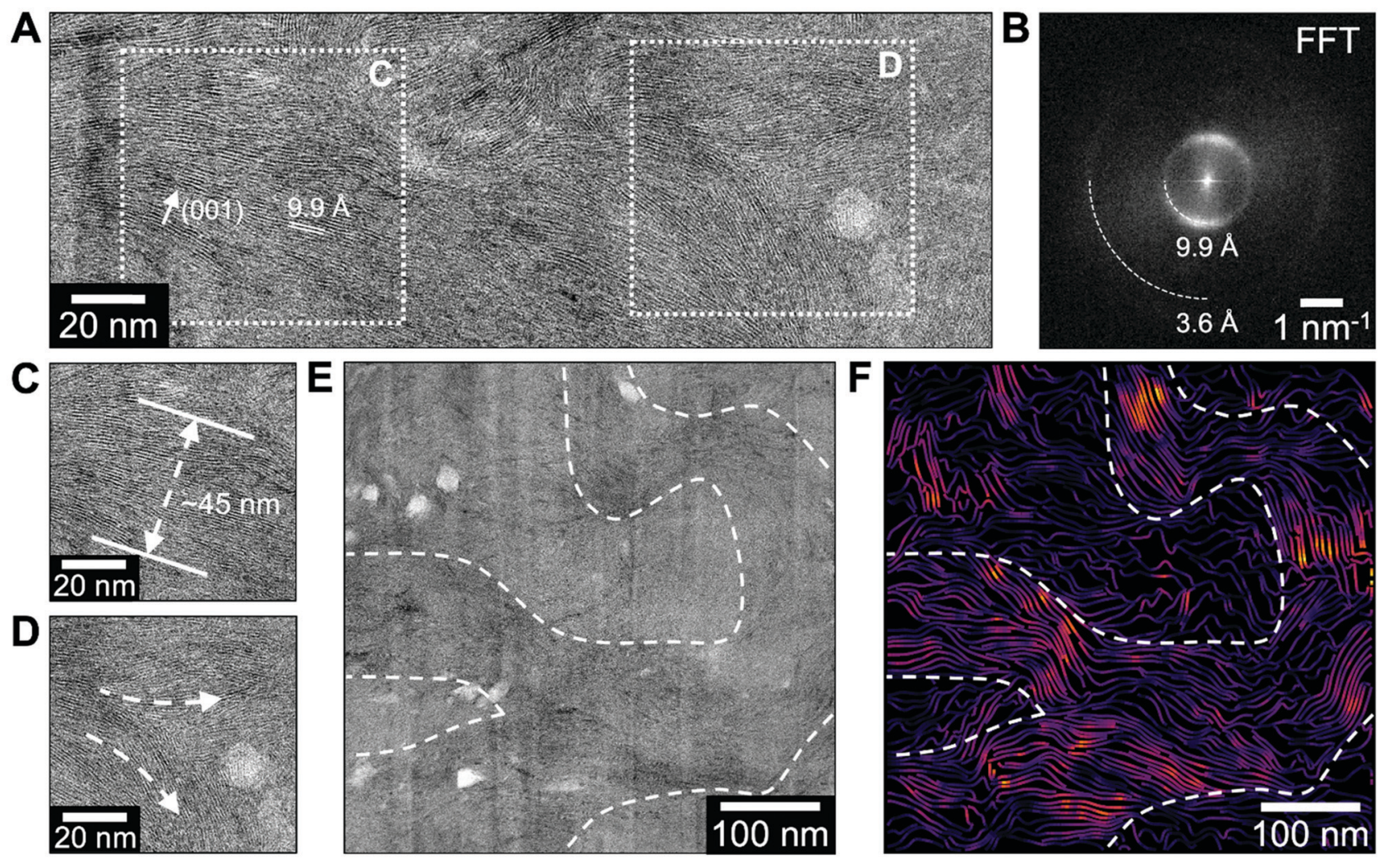

Fig. 4 High-resolution cross-sectional STEM analysis of free-standing film flake structure. (A) BF-STEM imaging of flake cross-sections revealed large regions of continuous atomic-scale structure. (B) FFTs of large BF-STEM images covering hundreds of nanometers showed that layers generally orient to a consistent direction within the film, but with a distribution of local flake orientations. Local structural features were directly observed in BF-STEM images, such as (A) local flake thickness, here $\sim 45 \mathrm{~nm}$, and (B) locations where layers delaminate. (E) Bending of flakes over hundreds of nanometers was also observed in some regions, and (F) local layer orientation mapping was used to enhance visualization of these features, with line direction perpendicular to the local bilayer stacking direction and line brightness representing the lattice contrast in the corresponding BF-STEM image.

respectively. Additionally, film grinding prior to the XRD measurements could facilitate absorption of water from air causing expansion of the layers. Local structures can also be directly observed in the BF-STEM images, providing information about flake thickness, such as in Fig. 4C, or regions where flakes delaminate, such as in Fig. 4D, for example. In addition, imaging revealed that flakes can take on a curved geometry, as shown in Fig. 4E. This property is most clearly seen in Fig. $4 \mathrm{~F}$, where the local orientation of the flake lattice has been visualized in a stream plot, where the stream line direction is perpendicular to the bilayer stacking direction and stream line brightness corresponds to the magnitude of lattice contrast. Here, a single continuous flake $\sim 100 \mathrm{~nm}$ across contains vanadium bilayers that are oriented both vertically and horizontally within a few hundred nanometers of each other. This observation indicates that the exfoliated flakes are able to bend without compromising the bilayer structure. Based on the combination of SEM and STEM imaging, the probe sonicationbased exfoliation technique results in conversion of crumpled sheets to 2D hierarchically arranged straightened nanoflakes. These flakes, arranged in ordered and flexible stacks, should therefore provide enhanced electron transport that can lead to potential applications as electrodes for electrochemical energy storage.

To confirm whether the material does indeed have enhanced electron transport, we performed conductivity measurements, summarized in Table 1. The measurements showed that the conductivity of the free-standing film is an order of magnitude higher than that of the pellets prepared by pressing precursor powder. For example, precursor powder samples have individual conductivities of $8.7 \times 10^{-5}$ (sample A) and $6.0 \times 10^{-5} \mathrm{~S} \mathrm{~cm}^{-1}$ (sample B), and the film, prepared by vacuum filtration of mixed suspensions prepared by exfoliation of samples A and B, exhibited a conductivity of $4.2 \times 10^{-4} \mathrm{~S} \mathrm{~cm}^{-1}$ (Table 1). The higher conductivity values of the film can be attributed to the formation of a more uniform and homogenous

Table 1 Four-probe conductivity measurement results obtained on pellets made by pressing the $\delta-\mathrm{Li}_{x} \mathrm{~V}_{2} \mathrm{O}_{5} \cdot n \mathrm{H}_{2} \mathrm{O}$ precursor powder and corresponding films prepared by vacuum filtration of the exfoliated precursor in NMP. Precursors A and B were used to prepare film 1; precursors C and D were used to prepare film 2; and precursor E was used to prepare film 3

\begin{tabular}{|c|c|c|c|c|c|}
\hline $\begin{array}{l}\text { Precursor } \\
\text { sample ID }\end{array}$ & $\begin{array}{l}\text { Precursor } \\
\text { pellet } \\
\text { thickness } \\
(\mathrm{mm})\end{array}$ & $\begin{array}{l}\text { Precursor } \\
\text { conductivity } \\
\left(\mathrm{S} \mathrm{cm}^{-1}\right)\end{array}$ & $\begin{array}{l}\text { Film } \\
\text { sample ID }\end{array}$ & $\begin{array}{l}\text { Film } \\
\text { thickness } \\
(\mathrm{mm})\end{array}$ & $\begin{array}{l}\text { Film } \\
\text { conductivity } \\
\left(\mathrm{S} \mathrm{cm}^{-1}\right)\end{array}$ \\
\hline A & 0.013 & $8.7 \times 10^{-5}$ & $1(\mathrm{~A}+\mathrm{B})$ & 0.0035 & $4.2 \times 10^{-4}$ \\
\hline B & 0.022 & $6.0 \times 10^{-5}$ & & & \\
\hline $\mathrm{C}$ & 0.021 & $4.2 \times 10^{-5}$ & $2(C+D)$ & 0.0014 & $3.5 \times 10^{-4}$ \\
\hline D & 0.096 & $3.6 \times 10^{-5}$ & & & \\
\hline $\mathrm{E}$ & 0.115 & $6.4 \times 10^{-6}$ & $3(\mathrm{E})$ & 0.0042 & $4.0 \times 10^{-4}$ \\
\hline
\end{tabular}


layers upon exfoliation with fewer boundaries in lateral space. Additionally, partial reduction of vanadium leading to the mixed vanadium oxidation state of $\mathrm{V}^{4+} / \mathrm{V}^{5+}$ in the film could contribute to the increase in measured conductivity values. ${ }^{45}$ To confirm this result, multiple batches of precursor powders and films were tested, and each film showed a similar increase in conductivity. There are more precursor data points because multiple precursor samples were frequently combined to create a film.

\section{Conclusion}

This work demonstrates successful exfoliation of bilayered $\delta-\mathrm{Li}_{x} \mathrm{~V}_{2} \mathrm{O}_{5} \cdot n \mathrm{H}_{2} \mathrm{O}$ powder for the first time. Bulk precursor was prepared using a low-temperature sol-gel process involving through-synthesis insertion of $\mathrm{Li}^{+}$ions into the interlayer region of the growing layered oxide phase, called chemical preintercalation. Exfoliation was achieved using a simple probe sonication technique in NMP solvent. Subsequently, freestanding films were fabricated by vacuum filtration of the produced suspensions. Structural and chemical characterization showed that the exfoliation process did not result in phase degradation or formation of secondary impurity phases. XRD patterns demonstrated a slightly smaller interlayer spacing for the exfoliated sample than its bulk counterpart, which has been attributed to the restacking and changes in the interlayer region chemistry. The exfoliation process resulted in a change of morphology from thick crumpled sheets to stacked nanoflakes. This change in morphology likely assisted in the enhanced electronic conductivity of free-standing films $\left(\sim 10^{-4} \mathrm{~S} \mathrm{~cm}^{-1}\right)$, which is an order of magnitude higher than that of the precursor powder $\left(\sim 10^{-5} \mathrm{~S} \mathrm{~cm}^{-1}\right)$.

The produced films can be utilized in a number of research applications, most notably as a "free-standing" electrode in metal-ion rechargeable battery systems. The increased conductivity is especially important for electrochemical energy storage where high electronic conductivities are important for fast charge and discharge applications. This top-down exfoliation technique provides an important path forward to develop exfoliated layers, which can serve as a key component of $2 \mathrm{D}$ heterostructures. Lastly, this result could be adapted for the exfoliation of other layered hydrated metal oxides with expanded interlayer regions such as titanates ${ }^{32}$ or tungsten oxides. $^{46}$

\section{Author contributions}

E. P. developed the concept and designed the experiments. L. H. carried out synthesis including exfoliation and free-standing film formation experiments. S. M. performed XRD characterization and supervised L. $\mathrm{H}$. in running electronic conductivity measurements. R. A. carried out XPS characterization. M. Z. performed STEM imaging and image analysis. All authors contributed to the interpretation of obtained experimental data and writing of the manuscript.

\section{Conflicts of interest}

There are no conflicts to declare.

\section{Acknowledgements}

This work was supported by the National Science Foundation under Grant No. DMR-1752623 and Leading Foreign Research Institute Recruitment Program funded by the Korean National Research Foundation (NRF) via the NNFC-KAIST-Drexel-SMU FIRST Nano ${ }^{2}$ Co-op Center (2016K1A4A3945038, 2015K1A4A3047100). XRD characterization and conductivity measurements were supported and as part of the Center for Mesoscale Transport Properties, an Energy Frontier Research Center supported by the U.S. Department of Energy, Office of Science, Basic Energy Sciences, under award \#DE-SC0012673. Scanning transmission electron microscopy was conducted at the Center for Nanophase Materials Sciences, which is a DOE Office of Science User Facility. We thank Drexel's Materials Characterization Core (MCC) facility for providing access to characterization instruments.

\section{References}

1 K. S. Novoselov, A. Mishchenko, A. Carvalho and A. H. Castro, Neto, 2D materials and van der Waals heterostructures., Science, 2016, 353(6298), aac9439.

2 S. Mukherjee and G. Singh, Two-Dimensional Anode Materials for Non-lithium Metal-Ion Batteries, ACS Appl. Energy Mater., 2019, 2(2), 932-955.

3 H. Zhang, M. Chhowalla and Z. Liu, 2D nanomaterials: graphene and transition metal dichalcogenides, Chem. Soc. Rev., 2018, 47(9), 3015-3017.

4 B. Anasori, M. R. Lukatskaya and Y. Gogotsi, 2D metal carbides and nitrides (MXenes) for energy storage. Nature Reviews, Materials, 2017, 2(2), 16098.

5 A. Gupta, T. Sakthivel and S. Seal, Recent development in 2D materials beyond graphene, Prog. Mater. Sci., 2015, 73, 44-126.

6 R. Mas-Ballesté, C. Gómez-Navarro, J. Gómez-Herrero and F. Zamora, 2D materials: to graphene and beyond, Nanoscale, 2011, 3(1), 20-30.

7 S. Mukherjee, Z. Ren and G. Singh, Beyond Graphene Anode Materials for Emerging Metal Ion Batteries and Supercapacitors, Nano-Micro Lett., 2018, 10(4), 70.

8 X. Zhang, L. Hou, A. Ciesielski and P. Samorì, 2D Materials Beyond Graphene for High-Performance Energy Storage Applications, Adv. Energy Mater., 2016, 6(23), 1600671.

9 R. Sahoo, A. Pal and T. Pal, 2D materials for renewable energy storage devices: Outlook and challenges, Chem. Commun., 2016, 52(93), 13528-13542.

10 H. Tang, Q. Hu, M. Zheng, Y. Chi, X. Qin, H. Pang and Q. Xu, MXene-2D layered electrode materials for energy storage, Prog. Nat. Sci.: Mater. Int., 2018, 28(2), 133-147.

11 Y. Jiao, A. M. Hafez, D. Cao, A. Mukhopadhyay, Y. Ma and H. Zhu, Metallic $\mathrm{MoS}_{2}$ for High Performance Energy Storage and Energy Conversion., Small, 2018, 14(36), 1800640. 
12 V. Nicolosi, M. Chhowalla, M. G. Kanatzidis, M. S. Strano and J. N. Coleman, Liquid Exfoliation of Layered Materials, Science, 2013, 340(6139), 1226419.

13 J. N. Coleman, M. Lotya, A. O'Neill, S. D. Bergin, P. J. King, U. Khan, K. Young, A. Gaucher, S. De, R. J. Smith, I. V. Shvets, S. K. Arora, G. Stanton, H.-Y. Kim, K. Lee, G. T. Kim, G. S. Duesberg, T. Hallam, J. J. Boland, J. J. Wang, J. F. Donegan, J. C. Grunlan, G. Moriarty, A. Shmeliov, R. J. Nicholls, J. M. Perkins, E. M. Grieveson, K. Theuwissen, D. W. McComb, P. D. Nellist and V. Nicolosi, Two-Dimensional Nanosheets Produced by Liquid Exfoliation of Layered Materials, Science, 2011, 331(6017), 568.

14 W. M. R. Divigalpitiya, R. F. Frindt and S. R. Morrison, Inclusion Systems of Organic Molecules in Restacked Single-Layer Molybdenum Disulfide, Science, 1989, 246(4928), 369.

15 X. Fan, P. Xu, Y. C. Li, D. Zhou, Y. Sun, M. A. T. Nguyen, M. Terrones and T. E. Mallouk, Controlled Exfoliation of $\mathrm{MoS}_{2}$ Crystals into Trilayer Nanosheets., J. Am. Chem. Soc., 2016, 138(15), 5143-5149.

16 A. G. Kelly, V. Vega-Mayoral, J. B. Boland and J. N. Coleman, Whiskey-phase exfoliation: exfoliation and printing of nanosheets using Irish whiskey, 2D Mater., 2019, 6(4), 045036.

17 C. Zhao, X. Wang, J. Kong, J. M. Ang, P. S. Lee, Z. Liu and X. Lu, Self-Assembly-Induced Alternately Stacked SingleLayer MoS2 and N-doped Graphene: A Novel van der Waals Heterostructure for Lithium-Ion Batteries, ACS Appl. Mater. Interfaces, 2016, 8(3), 2372-2379.

18 W. Liu, Z. Wang, Y. Su, Q. Li, Z. Zhao and F. Geng, Molecularly Stacking Manganese Dioxide/Titanium Carbide Sheets to Produce Highly Flexible and Conductive Film Electrodes with Improved Pseudocapacitive Performances, Adv. Energy Mater., 2017, 7(22), 1602834.

19 W. Tian, A. VahidMohammadi, Z. Wang, L. Ouyang, M. Beidaghi and M. M. Hamedi, Layer-by-layer selfassembly of pillared two-dimensional multilayers, Nat. Commun., 2019, 10(1), 2558.

20 V. Vega-Mayoral, R. Tian, A. G. Kelly, A. Griffin, A. Harvey, M. Borrelli, K. Nisi, C. Backes and J. N. Coleman, Solvent exfoliation stabilizes $\mathrm{TiS}_{2}$ nanosheets against oxidation, facilitating lithium storage applications., Nanoscale, 2019, 11(13), 6206-6216.

21 E. Varrla, C. Backes, K. R. Paton, A. Harvey, Z. Gholamvand, J. McCauley and J. N. Coleman, Large-Scale Production of Size-Controlled $\mathrm{MoS}_{2}$ Nanosheets by Shear Exfoliation., Chem. Mater., 2015, 27(3), 1129-1139.

22 E. Pomerantseva, F. Bonaccorso, X. Feng, Y. Cui and Y. Gogotsi, Energy storage: The future enabled by nanomaterials, Science, 2019, 366(6468), aan8285.

23 Y. Wang and G. Cao, Synthesis and Enhanced Intercalation Properties of Nanostructured Vanadium Oxides, Chem. Mater., 2006, 18(12), 2787-2804.

24 R. Baddour-Hadjean, L. N. T. Huynh, D. Batyrbekuly, S. Bach and J. P. Pereira-Ramos, Bilayered Potassium
Vanadate $\mathrm{K}_{0.5} \mathrm{~V}_{2} \mathrm{O}_{5}$ as Superior Cathode Material for NaIon Batteries, ChemSusChem, 2019, 12, 5192-5198.

25 M. Clites, B. W. Byles and E. Pomerantseva, Bilayered vanadium oxide as the host material for reversible beyond lithium ion intercalation, Adv. Mater. Lett., 2017, 8, 679-688.

26 M. Clites and E. Pomerantseva, Bilayered vanadium oxides by chemical pre-intercalation of alkali and alkali-earth ions as battery electrodes, Energy Storage Mater., 2018, 11, 30-37. 27 S. H. Lee, R. A. DiLeo, A. C. Marschilok, K. J. Takeuchi and E. S. Takeuchi, Sol Gel Based Synthesis and Electrochemistry of Magnesium Vanadium Oxide: A Promising Cathode Material for Secondary Magnesium Ion Batteries, ECS Electrochem. Lett., 2014, 3(8), A87-A90.

28 A. Moretti, G. Giuli, A. Trapananti and S. Passerini, Electrochemical and structural investigation of transition metal doped $\mathrm{V}_{2} \mathrm{O}_{5}$ sono-aerogel cathodes for lithium metal batteries., Solid State Ionics, 2018, 319, 46-52.

29 A. Moretti and S. Passerini, Bilayered Nanostructured $\mathrm{V}_{2} \mathrm{O}_{5}$. $\mathrm{nH}_{2} \mathrm{O}$ for Metal Batteries. Advanced Energy, Materials, 2016, 6(23), 1600868.

30 X. M. Xu, M. Y. Duan, Y. F. Yue, Q. Li, X. Zhang, L. Wu, P. J. Wu, B. Song and L. Q. Mai, Bilayered $\mathrm{Mg}_{0.25} \mathrm{~V}_{2} \mathrm{O}_{5} \cdot \mathrm{H}_{2} \mathrm{O}$ as a Stable Cathode for Rechargeable Ca-Ion Batteries, ACS Energy Lett., 2019, 4(6), 1328-1335.

31 V. Petkov, P. N. Trikalitis, E. S. Bozin, S. J. L. Billinge, T. Vogt and M. G. Kanatzidis, Structure of $\mathrm{V}_{2} \mathrm{O}_{5} \cdot \mathrm{nH}_{2} \mathrm{O}$ Xerogel Solved by the Atomic Pair Distribution Function Technique., J. Am. Chem. Soc., 2002, 124(34), 10157-10162.

32 S. Mukherjee, C. D. Quilty, S. Yao, C. A. Stackhouse, L. Wang, K. J. Takeuchi, E. S. Takeuchi, F. Wang, A. C. Marschilok and E. Pomerantseva, The effect of chemically preintercalated alkali ions on the structure of layered titanates and their electrochemistry in aqueous energy storage systems, J. Mater. Chem. A, 2020, 8(35), 18220-18231.

33 M. Clites, B. W. Byles and E. Pomerantseva, Effect of aging and hydrothermal treatment on electrochemical performance of chemically pre-intercalated $\mathrm{Na}-\mathrm{V}-\mathrm{O}$ nanowires for Na-ion batteries, J. Mater. Chem. A, 2016, 4(20), 7754-7761.

34 M. Clites, J. L. Hart, M. L. Taheri and E. Pomerantseva, Chemically Preintercalated Bilayered $\mathrm{K}_{\mathrm{x}} \mathrm{V}_{2} \mathrm{O}_{5} \cdot \mathrm{nH}_{2} \mathrm{O}$ Nanobelts as a High-Performing Cathode Material for K-Ion Batteries., ACS Energy Lett., 2018, 3(3), 562-567.

35 M. Clites, J. L. Hart, M. L. Taheri and E. Pomerantseva, Annealing-Assisted Enhancement of Electrochemical Stability of Na-Preintercalated Bilayered Vanadium Oxide Electrodes in Na-Ion Batteries, ACS Appl. Energy Mater., 2020, 3(1), 1063-1075.

36 C. Zhang, S.-H. Park, S. E. O’Brien, A. Seral-Ascaso, M. Liang, D. Hanlon, D. Krishnan, A. Crossley, N. McEvoy, J. N. Coleman and V. Nicolosi, Liquid exfoliation of interlayer spacing-tunable 2D vanadium oxide nanosheets: High capacity and rate handling Li-ion battery cathodes, Nano Energy, 2017, 39, 151-161. 
37 X. Rui, Z. Lu, H. Yu, D. Yang, H. H. Hng, T. M. Lim and Q. Yan, Ultrathin $\mathrm{V}_{2} \mathrm{O}_{5}$ nanosheet cathodes: realizing ultrafast reversible lithium storage., Nanoscale, 2013, 5(2), 556-560.

38 A. M. Diem, A. Knöller, Z. Burghard and J. Bill, Freestanding nanostructured vanadium pentoxide films for metal-ion batteries, Nanoscale, 2018, 10(33), 15736-15746.

39 A. S. Etman, A. K. Inge, X. Jiaru, R. Younesi, K. Edström and J. Sun, A Water Based Synthesis of Ultrathin Hydrated Vanadium Pentoxide Nanosheets for Lithium Battery Application: Free Standing Electrodes or Conventionally Casted Electrodes?, Electrochim. Acta, 2017, 252, 254-260.

40 A. S. Etman, H. D. Asfaw, N. Yuan, J. Li, Z. Zhou, F. Peng, I. Persson, X. Zou, T. Gustafsson, K. Edström and J. Sun, A one-step water based strategy for synthesizing hydrated vanadium pentoxide nanosheets from $\mathrm{VO}_{2}(\mathrm{~B})$ as freestanding electrodes for lithium battery applications, J. Mater. Chem. A, 2016, 4(46), 17988-18001.

41 E. Pomerantseva and Y. Gogotsi, Two-dimensional heterostructures for energy storage, Nat. Energy, 2017, 2(7), 17089.

42 C.-Y. Lee, A. C. Marschilok, A. Subramanian, K. J. Takeuchi and E. S. Takeuchi, Synthesis and characterization of sodium vanadium oxide gels: the effects of water (n) and sodium (x) content on the electrochemistry of $\mathrm{Na}_{\mathrm{x}} \mathrm{V}_{2} \mathrm{O}_{5}$. $\mathrm{nH}_{2}$ O., Phys. Chem. Chem. Phys., 2011, 13(40), 18047-18054.

43 M. Ghidiu, J. Halim, S. Kota, D. Bish, Y. Gogotsi and M. W. Barsoum, Ion-Exchange and Cation Solvation Reactions in $\mathrm{Ti}_{3} \mathrm{C}_{2}$ MXene., Chem. Mater., 2016, 28(10), 3507-3514.

44 E. A. Johnson and J. E. Post, Water in the interlayer region of birnessite: Importance in cation exchange and structural stability, Am. Mineral., 2006, 91(4), 609-618.

45 G. Manjunath, R. V. Vardhan, A. Salian, R. Jagannatha, M. Kedia and S. Mandal, Effect of annealing-temperatureassisted phase evolution on conductivity of solution combustion processed calcium vanadium oxide films, Bull. Mater. Sci., 2018, 41(5), 126.

46 J. B. Mitchell, N. R. Geise, A. R. Paterson, N. C. Osti, Y. Sun, S. Fleischmann, R. Zhang, L. A. Madsen, M. F. Toney, D.-E. Jiang, A. I. Kolesnikov, E. Mamontov and V. Augustyn, Confined Interlayer Water Promotes Structural Stability for High-Rate Electrochemical Proton Intercalation in Tungsten Oxide Hydrates., ACS Energy Lett., 2019, 4(12), 2805-2812. 